\title{
ECONOMIC EVALUATION OF IMIPENEM-CILASTATIN VERSUS DORIPENEM IN VENTILATOR- ASSOCIATED PNEUMONIA IN EGYPT
}

\author{
NERMEEN ASHOUSH ${ }^{1,2 *}$ \\ ${ }^{1}$ Department of Clinical Pharmacy and Pharmacy Practice, Faculty of Pharmacy, The British University in Egypt, El-Sherouk, Cairo 11837, \\ Egypt. ${ }^{2}$ Health Economics Unit, Center for Drug Research and Development (CDRD), The British University in Egypt, El-Sherouk, Cairo \\ 11837, Egypt. Email: nermeen.ashoush@bue.edu.eg \\ Received: 21 February 2017, Revised and Accepted: 22 February 2017
}

ABSTRACT

Objective: One of the most common complications of critical illness among surgical and trauma patients is ventilator-associated pneumonia (VAP). The mortality rate increases due to inappropriate empiric treatment of VAP. The rates of Pseudomonas aeruginosa (PA) VAP susceptibility to doripenem (DOR) are higher than those to imipenem-cilastatin (IMI). Cost-effectiveness analysis was developed to define outcome differences between strategies of empiric treatment of VAP with DOR versus IMI in Egyptian patients.

Methods: A cost-effectiveness model was designed comparing empiric treatment of VAP with DOR versus IMI from the health insurance perspectives. The differences in the total costs and quality-adjusted life years (QALYs) under each scenario were examined, and sensitivity analyses were conducted to determine the stability of our estimates. Drug costs were taken from health insurance hospitals, with other inputs derived from the literature.

Results: Deterministic baseline results from the model of DOR compared to IMI in the treatment of VAP suggest that DOR is more effective and less expensive than IMI. The total costs for IMI and DOR were Egyptian pound (EGP) 4646.93 and EGP 4197.58, respectively. QALYs for IMI and DOR were 0.53 and 0.54 , respectively.

Conclusions: Given the microbiologic sensitivity profile of PA to DOR and IMI, and depending on the local susceptibility patterns in institutions where DOR in vitro susceptibilities are superior to those of other carbapenems for PA clinical isolates, empiric treatment of VAP with DOR may dominate that with IMI by being both life- and cost-saving.

Keywords: Ventilator-associated pneumonia, Cost-effectiveness, Doripenem, Imipenem-cilastatin.

(c) 2017 The Authors. Published by Innovare Academic Sciences Pvt Ltd. This is an open access article under the CC BY license (http://creativecommons. org/licenses/by/4. 0/) DOI: http://dx.doi.org/10.22159/ajpcr.2017.v10i6.18066

\section{INTRODUCTION}

Ventilator-associated pneumonia (VAP) means nosocomial pneumonia occurring 48 hrs or more after initiation of mechanical ventilation (MV) [1]. VAP is the most common Hospital-Associated Infection among adult patients in intensive care units (ICUs), with frequencies ranging from $15 \%$ to $45 \%$ [2]. Increase in hospital morbidity, mortality, duration of hospitalization by an average of 7-9 days per patient, and increase in health-care costs were associated with VAP $[3,4]$. The incidence rates of VAP are higher in developing countries with limited resources [5].

The pathogenesis of VAP involves bacterial invasion of the pulmonary parenchyma in patients receiving MV. Inoculation of the previously sterile lower respiratory tract results from aspiration of secretions, colonization of the aerodigestive tract, or use of contaminated equipment or medications [6].

VAP is caused by such resistant organisms as methicillin-resistant Staphylococcus aureus and Pseudomonas aeruginosa (PA) [7]. Hence, because inappropriate empiric therapy for VAP leads to a higher hospital mortality rate $[8,9]$, broad coverage for such pathogens, followed by deescalation, is an evidence-based recommendation.

Imipenem/cilastatin (IMI) is a broad spectrum antibiotic possessing activity against clinically important aerobic Gram-positive, Gramnegative species, and anaerobes [10]. IMI is commonly used and recommended first-line therapies for hospital acquired pneumonia and VAP in patients at high risk for resistant pathogens. Recently, PA resistance to IMI has become an increasing challenge in VAP treatment.
Doripenem (DOR) is a novel, broad-spectrum parenteral carbapenem antimicrobial. It shows an enhanced in vitro potency against PA and a more favorable sensitivity pattern among PA isolates [11]. Because of these more favorable susceptibility patterns, empiric DOR use when IMI resistance is likely may improve both clinical and resource utilization outcomes.

The objective of this study was to evaluate the cost-effectiveness of IMI versus DOR in VAP from the health insurance perspective over a time horizon of 15 days.

\section{METHODS}

It is important to identify the most cost-effective treatment in patients with VAP. Decision analysis is a quantitative method for synthesizing data from numerous sources for the evaluation of treatment alternatives and was developed to determine the cost-effectiveness of the IMI strategy, as compared to DOR in patients with VAP.

The literature search was conducted in Medline, PubMed, and Cochrane Library to identify relevant published English articles from January 2000 to October 2016. The decision analytical model was constructed to assess the costs and consequences associated with IMI compared with DOR. The clinical parameters were derived mainly from two sources; the first was a prospective, multicenter, parallel randomized, active-controlled, open-label study that compares DOR versus IMI on 531 patients with VAP. The model structure was derived from the costeffectiveness model of empiric DOR compared with IMI in VAP in the United States [12,13] 
The utilities were derived from two sources; the first was a cost-utility analysis that used Markov model to compare meropenem with IMI in the treatment of severe infections in intensive care. The study elicits the utility scores from the EuroQoL (EQ-5D) social tariff for "unconscious" patients in the United Kingdom [14]. The second source was a costeffectiveness model of empiric DOR compared with IMI in VAP that used utilities estimated using time-trade off questions from 1005 patients enrolled in a five-center study of seriously ill patients [13].

This is an analysis of publicly accessible data; no human subjects were enrolled in the study. All calculations were performed in Excel 2016. The target population is Egyptian patients in the health-care system, no subgroup analysis. Total costs include costs of treatment and managing strategies according to the Egyptian current practice. The source of costs was the hospitals. The outcomes of the two treatments were measured in terms of quality-adjusted life-years (QALYs). The method used; EQ5D from published literature. All lives, life years, or QALYs are valued equally, regardless of age, gender, or socioeconomic status of individuals in the population. Critical component (s) in the calculation is varied through a relevant range or from worst case to best case. One-way sensitivity analysis is performed.

\section{Model overview and structure}

A cost-effectiveness model was developed of DOR versus IMI as the initial empiric antibiotic for VAP in patients on MV. In a decision analysis approach, a single decision node represented the choice to treat VAP with DOR or IMI in patients on MV. This cohort was marched through each branch of the decision tree to either death or VAP resolution and calculated the incremental costs associated with each choice (Fig. 1).

The parameters for the incidence of PA-VAP and its clinical and economic consequences were derived from a review of literature and other publicly accessible data (Table 1). We defined cost-effectiveness from the health insurance perspective as the ratio between total hospital cost differences and the differences in QALY between the two strategies.

Because of the uncertainty surrounding various model parameters, sensitivity analyses was performed to assess the precision and stability of the observations. The 95\% confidence intervals (CIs) around the point estimates were calculated.

\section{Patterns of DOR and IMI susceptibility among PA}

In a microbiology registry of 270PA isolates from patients in the ICU, $220(81.5 \%)$ were DOR sensitive (DOR-S); of those, 21 (7.8\%) were also IMI resistant (IMI-R) and $50(18.5 \%)$ were DOR-R, with the rate of IMI-S in this group of $1(0.4 \%)$ [12]. Drug sensitivity was defined on the basis of the current U.S. Food and Drug Administration thresholds for each drug: For IMI, a minimum inhibitory concentration (MIC)

Table 1: Model inputs

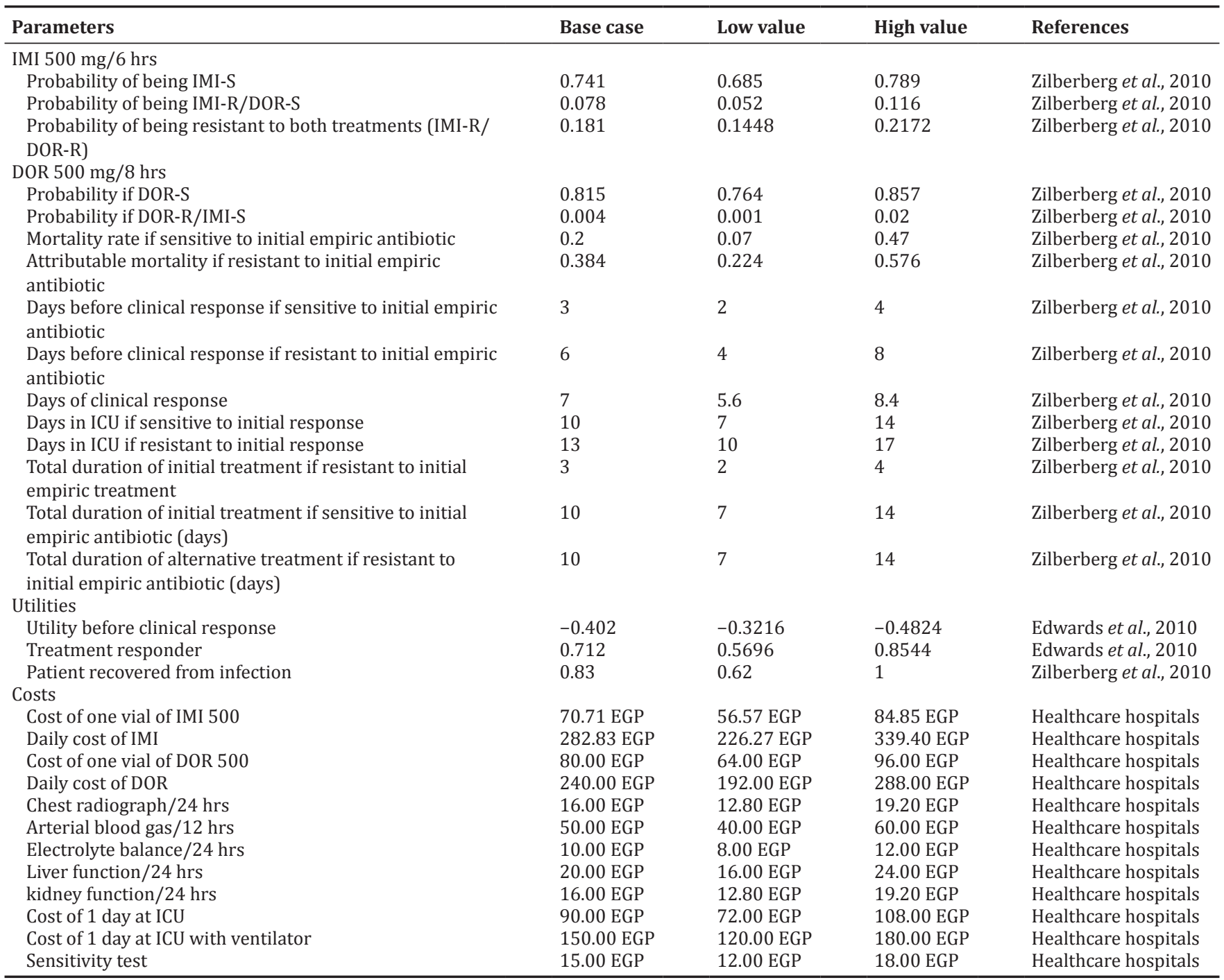

IMI-S: Imipenem-cilastatin sensitive, DOR-S: Doripenem sensitive, IMI-R: Imipenem-cilastatin resistant, DOR-R: Doripenem resistant, EGP: Egyptian pound,

ICU: Intensive care unit 


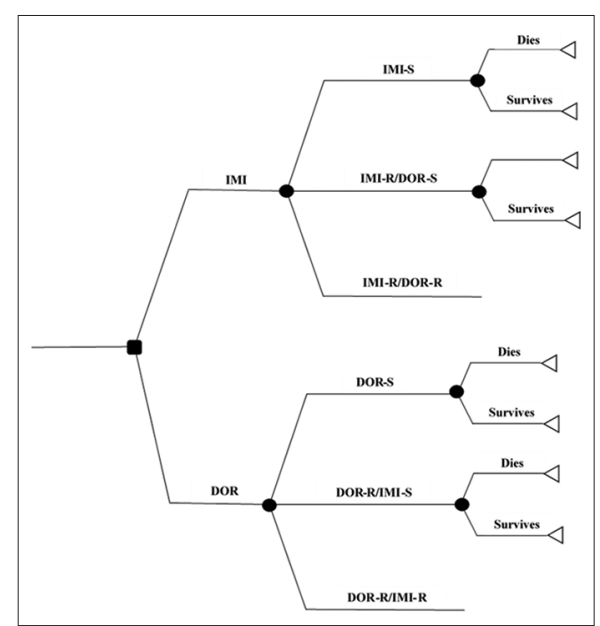

Fig. 1: Decision tree. Square node at left represents the decision to treat empirically with DOR or IMI. Circular chance nodes represent the probability of the various options for susceptibility of PA. The final circular event node represents the outcome: Death or survival. Rightmost vertical segments are terminal nodes. DOR: Doripenem, IMI: Imipenem-cilastatin, R: Resistant; S: Sensitive.

of $\leq 4 \mu \mathrm{g} / \mathrm{mL}$ is susceptible, eight $\mu \mathrm{g} / \mathrm{mL}$ is intermediate, and $\geq 16 \mu \mathrm{g} / \mathrm{mL}$ is resistant. For DOR, a MIC of $\leq 2 \mu \mathrm{g} / \mathrm{mL}$ is susceptible and $\geq 4 \mu \mathrm{g} / \mathrm{mL}$ is nonsusceptible [11].

According to these data, the rates of susceptibility utilized in the model were as follows: DOR-S 0.815 (95\% CI: 0.764, 0.857), IMI-S 0.741 (95\% CI: $0.685,0.789$ ), DOR-R/IMI-S 0.004 (95\% CI: 0.001, 0.020), and IMI-R/ DOR-S 0.078 (95\% CI: $0.052,0.116)$. Although the point estimates for each parameter were used in the base case of the model, each estimate was varied along its corresponding 95\% CI in the sensitivity analyses.

Attributable mortality rate and mortality rate reduction with appropriate treatment

The estimates for PA attributable deaths were reported by Brewer et al. [15]. In this single-center study of 38 consecutive PA-VAP patients, the PA-VAP-attributable mortality rate was $38.4 \%$ [15]. The mortality rate reduction conferred was estimated by timely institution of an appropriate therapy on the basis of a study by Edwards et al. [16], where the relative risk of death among patients with IMI-R versus IMI-S PA infections was 1.86 (95\% CI: 1.38, 2.51) [16]. To calculate the mortality rate reduction attributable to appropriate empiric therapy of PA-VAP, this estimate was inverted.

\section{Duration, dosage, and costs of treatment}

According to the guidelines from the American Thoracic Society (ATS) and the Infectious Diseases Society of America (IDSA), a 10-day IMI treatment course was used in the base case for two reasons: (1) The guideline is vague on the recommended duration of treatment and (2) assuming a traditional 14-day course, reduction to 10 days represents an approximate adjustment for the fact that some patients die before completing their treatment [10]. The duration and dosage of DOR were based on the Phase III trial for this drug with a similar adjustment for early death [12]. This parameter was varied between 7 and 14 days for each strategy.

The daily IMI dose was $500 \mathrm{mg} \mathrm{q} 6 \mathrm{hrs}$ and that for DOR $500 \mathrm{mg} \mathrm{q} 8 \mathrm{hrs}$ and was validated by an expert opinion. In the base case, a 3-day lag period between the onset of VAP and the availability of lower airway culture and sensitivity results was assumed, and was varied between 2 and 4 days.

The patients treated with DOR testing positive for DOR-R/IMI-S PA were switched to IMI for an additional 10 days (range 7-14 days), and those treated with IMI testing positive for IMI-R/DOR-S PA were switched to DOR for an additional 10-day course (range 7-14 days). If a specimen tested positive for a PA sensitive to the initial empiric antibiotic, this antibiotic was continued for the full 10-day course (range 7-14 days).

\section{Utilities and QALY}

QALYs capture the benefits of an intervention in terms of survival and health-related quality of life (HRQOL). QALYs are calculated by multiplying the length of time affected by an intervention by the HRQoL or utility experienced during that time, where utility is measured on a scale from 0 (dead) to 1 (perfect health). A single year of perfect health would equal " 1 " while a single year at only half the level of perfect health would equal " 0.5 " [14]. Table 1 shows the daily proportion of a QALY attached to each of the health states within the model. The pragmatic decision was therefore made to use the utility score from the EuroQoL (EQ-5D) social tariff for "unconscious" (P. Kind, G. Hardman and S. Macran, unpublished).

\section{RESULTS}

Deterministic baseline results from the model of DOR compared to IMI in the treatment of VAP suggest that DOR is more effective and less expensive than IMI (Table 2). Direct medical costs were obtained from the healthcare hospitals in Egypt. Total costs for IMI and DOR were Egyptian pound (EGP) 4646.93 and EGP 4197.58, respectively. QALYs for IMI and DOR were 0.525222843 and 0.536769973 , respectively. The incremental cost-effectiveness ratio for IMI versus DOR was L.E-38914.30/QALY (Table 3). This study showed that IMI is less effective and more costly compared to DOR in treating VAP patients.

As in all modeling exercises, several assumptions were made in this study leading to uncertainties in the results. In this analysis, it was explicitly accounted for these uncertainties by assigning CIs and plausible ranges based on published sources. To assess the influences of other model structures and assumptions on the cost-effectiveness estimates, one-way sensitivity analyses of various parameters were performed and showed that probability that patients are resistant to imipenem but sensitive to DOR (IMI-R/DOR-S), daily cost of IMI and DOR had the greatest impact on the results (Fig. 2).

\section{DISCUSSION}

This cost-effectiveness analysis shows that patients on MV who are suspected to have VAP, empiric treatment with DOR are not only cost-effective but cost saving when compared with treatment with IMI. Furthermore, as a hospital cost- and life-saving approach, DOR dominates the alternative of empiric IMI.

VAP remains a challenge in the ICU in general and even more so in surgical and trauma ICUs [17]. As it is caused by a resistant organism [18], and inappropriate empiric treatment of VAP results in a higher hospital mortality rate $[19,20]$, there is a challenge in the choice of VAP treatment. To balance the benefit to the patient of broad initial coverage against the risk of further antimicrobial resistance development, the joint ATSIDSA guidelines highlight the need for broad initial coverage followed by de-escalation to the extent possible according to lowerairway sampling [10]. At the same time, the value proposition for the advocated broad coverage needs to be made explicitly, given the higher costs of the newer broader-spectrum antibiotics than the older agents. This study builds on previous work investigating clinical and microbiologic comparisons between DOR and IMI by demonstrating cost savings with a DOR strategy over IMI as a treatment for suspected VAP, not only in the pharmacy acquisition costs but also in the overall hospital costs. By showing consistent hospital cost savings associated with DOR treatment, our results allow clinicians to focus on improving clinical outcomes for their patients with suspected VAP.

The main strengths of the study are the use of evidence from prospective, multicenter, parallel randomized study, and incorporating quality of life issues in clinical decisions. The study has a number of 


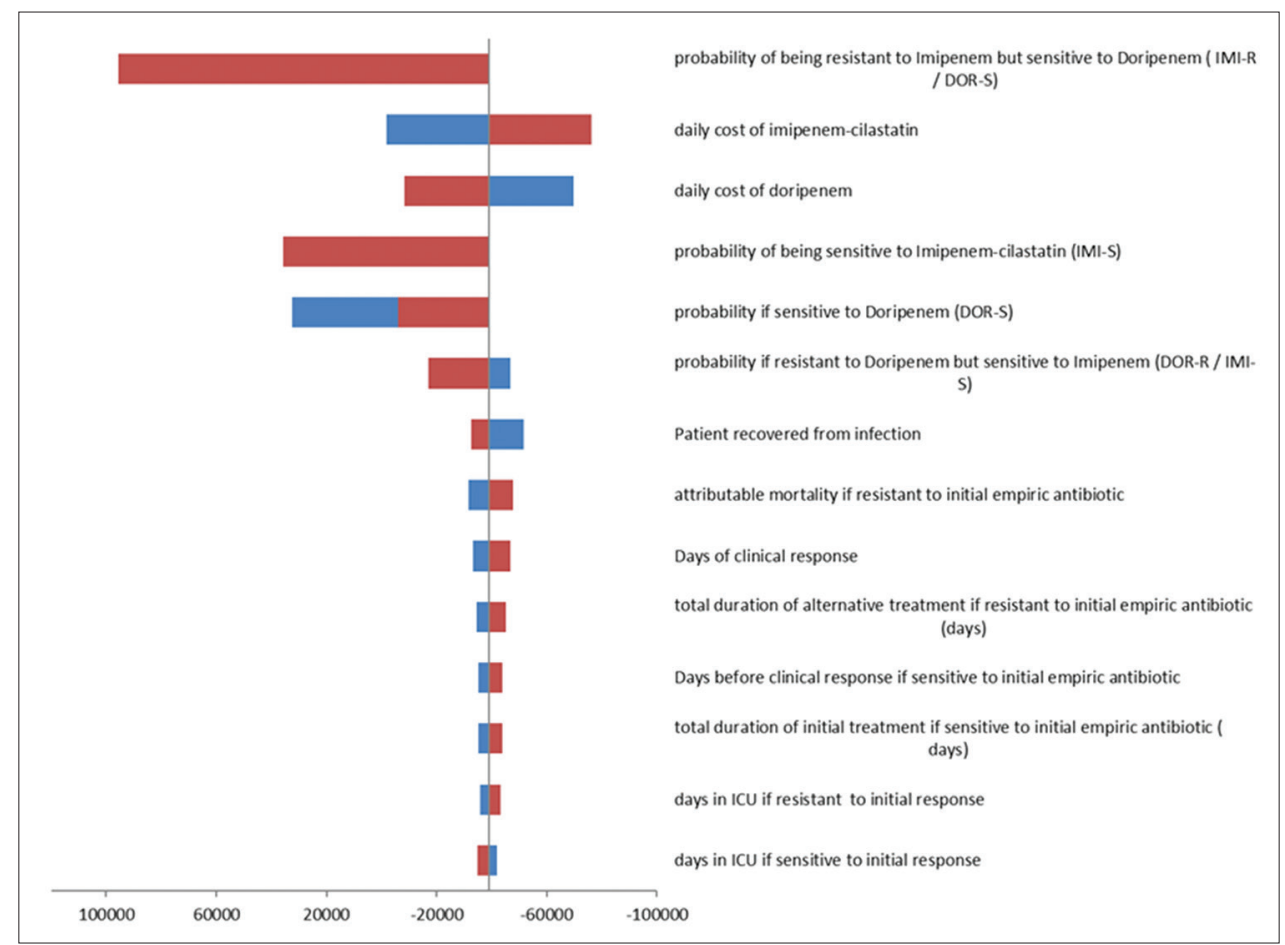

Fig. 2: Tornado diagram of costs and outcomes

Table 2: Outcomes of empiric treatment with DOR compared with IMI

\begin{tabular}{llllll}
\hline Parameters & Probabilities & Cost & Utility & Total cost & Total QALY \\
\hline IMI & & & & & \\
$\quad$ IMI-S & 0.741 & $5,543.32$ EGP & 0.010350685 & 821.52 EGP & 0.001534 \\
Dies & 0.2 & $5,543.32$ EGP & 0.817610959 & $3,286.08$ EGP & 0.48468 \\
Survives & 0.8 & & & & \\
IMI-R/DOR-S & 0.078 & $6,914.50$ EGP & 0.007046575 & 207.10 EGP & 0.000211 \\
Dies & 0.384 & $6,914.50$ EGP & 0.807484932 & 332.23 EGP & 0.038798 \\
Survives & 0.616 & & & $4,646.93$ EGP & 0.525222843 \\
DOR & & & & \\
DOR-S & 0.815 & & & & \\
Dies & 0.2 & $5,115.00$ EGP & 0.010350685 & & 0.001687 \\
Survives & 0.8 & $5,115.00$ EGP & 0.817610959 & $3,334.98$ EGP & 0.533082 \\
DOR-R/IMI-S & 0.004 & & & & \\
Dies & 0.384 & $7,214.32$ EGP & 0.007046575 & 11.08 EGP & 0.000011 \\
Survives & 0.616 & $7,214.32$ EGP & 0.807484932 & & 0.00199 \\
& & & & $4,197.58$ EGP & 0.536769973 \\
\hline
\end{tabular}

IMI-S: Imipenem-cilastatin sensitive, DOR-S: Doripenem sensitive, IMI-R: Imipenem-cilastatin resistant, DOR-R: Doripenem resistant, EGP: Egyptian pound, QALY: Quality-adjusted life years

\section{Table 3: Calculation of incremental cost effectiveness ration}

\begin{tabular}{ll}
\hline Inputs & Values \\
\hline$\Delta$ Cost (IMI-DOR) & 449.35 EGP \\
$\Delta$ QALY (IMI-DOR) & -0.01154713 \\
ICER for IMI versus DOR & $-38,914.30 / Q A L Y$ \\
$\Delta$ Cost $/ \triangle Q A L Y$ &
\end{tabular}

$\Delta$ Cost $_{\text {(IMLDOR) }} / \triangle \mathrm{QALY}_{\text {(IMI-DOR }}$

IMI: Imipenem-cilastatin, DOR: Doripenem, ICER: Incremental

cost-effectiveness ration, EGP: Egyptian pound, QALY: Quality-adjusted life years

limitations. First, the study was limited to PA patients as it is one of the leading pathogens in VAP. Second, because of the paucity of long-term data on VAP survivor published data on utilities in ICU so the utility score from the EuroQoL (EQ-5D) social tariff was used and it has been used in the previous studies $[13,14]$.

In summary, the results from this study showed that IMI is less effective and more costly compared to DOR in the treatment of VAP. These findings will help to inform health-care decision makers regarding the allocation of health-care system resources to improve the health of the Egyptian population.

\section{REFERENCES}

1. Liu B, Li SQ, Zhang SM, Xu P, Zhang X, Zhang YH, et al. Risk factors of ventilator associated pneumonia in a paediatric intensive care unit: A systematic review and meta-analysis. J Thorac Dis 2013;5(4):525-31.

2. Jaimes F, De La Rosa G, Gomez E, Munera P, Ramirez J, Castrillon S. Incidence and risk factors for ventilator-associated pneumonia in a developing country: Where is the difference? Respir Med 2007;101(4):762-7.

3. Urrea M, Pons M, Serra M, Latorre C, Palomeque A. Prospective incidence study of nosocomial infections in a pediatric intensive care unit. Pediatr Infect Dis J 2003;22(6):490-4.

4. Srinivasan R, Asselin J, Gildengorin G, Wiener-Kronish J, Flori HR. A prospective study of ventilator-associated pneumonia in children. Pediatrics 2009;123(4):1108-15.

5. Raza MW, Kazi BM, Mustafa M, Gould FK. Developing countries have their own characteristic problems with infection control. J Hosp Infect 
2004;57(4):294-9.

6. Abdel-Gawad TA, El-Hodhod MA, Ibrahim HM, Michael YW. Gastroesophageal reflux in mechanically ventilated paediatric patients and its relation to ventilator-associated pneumonia. Crit Care 2009; $13:$ R164

7. Chastre J, Fagon JY. Ventilator-associated pneumonia. Am J Respir Crit Care Med 2002; 165(7):867-903.

8. Alvarez-Lerma F. Modification of empiric antibiotic treatment in patients with pneumonia acquired in the intensive care unit. ICU-acquired pneumonia study group. Intensive Care Med 1996;22(5):387-94.

9. Iregui M, Ward S, Sherman G, Fraser VJ, Kollef MH. Clinical importance of delays in the initiation of appropriate antibiotic treatment for ventilator-associated pneumonia. Chest 2002;122(1):262-8.

10. Sheikh-Taha M, Khoury T, Chatila M. Assessment of the appropriate utilization of impinem/cilastatin in a tertiary care hospital. Int J Pharm Pharm Sci 2011;3(4):381-3.

11. Jayapraba S, Suresh A, Niraimathi V. Forced oxidative degradation study of doripenem by UV spectrophotometric method. Int J Pharm Pharm Sci 2013;5(1):430-1.

12. American Thoracic Society; Infectious Diseases Society of America. Guidelines for the management of adults with hospital-acquired, ventilator-associated, and healthcare-associated pneumonia. Am J Respir Crit Care Med 2005;171(4):388-416.

13. Brown N, Draghi DH, Yee YC, Evangelista AT, Sahm DF. Susceptibility of Pseudomonas aeruginosa to Doripenem and Imipenem in the United States: 2006-2007 TRUST Surveillance. Poster Presentation at the $37^{\text {th }}$ Critical Care Congress. Honolulu, HI, February, 2-6; 2008.

14. Chastre J, Wunderink R, Prokocimer P, Lee M, Kaniga K, Friedland I. Efficacy and safety of intravenous infusion of doripenem versus imipenem in ventilator-associated pneumonia: A multicenter, randomized study. Crit Care Med 2008;36(4):1089-96.

15. Zilberberg MD, Mody SH, Chen J, Shorr AF. Cost-effectiveness model of empiric doripenem compared with imipenem-cilastatin in ventilatorassociated pneumonia. Surg Infect (Larchmt) 2010;11(5):409-17.

16. Edwards SJ, Campbell HE, Plumb JM. Cost-utility analysis comparing meropenem with imipenem plus cilastatin in the treatment of severe infections in intensive care. Eur J Health Econ 2006;7(1):72-8.

17. Brewer SC, Wunderink RG, Jones CB, Leeper KV. Ventilatorassociated pneumonia due to Pseudomonas aeruginosa. Chest 1996;109:1019-29.

18. Lautenbach E, Weiner MG, Nachamkin I, Bilker WB, Sheridan A, Fishman NO. Imipenem resistance among pseudomonas aeruginosa isolates: Risk factors for infection and impact of resistance on clinical and economic outcomes. Infect Control Hosp Epidemiol 2006;27(9):893-900.

19. NNIS System. National nosocomial infections surveillance (NNIS) system report, data summary from January 1992 through June 2003, issued August 2003. Am J Infect Control 2003;31(8):481-98.

20. Warren DK, Shukla SJ, Olsen MA, Kollef MH, Hollenbeak CS, Cox MJ, et al. Outcome and attributable cost of ventilator-associated pneumonia among intensive care unit patients in a suburban medical center. Crit Care Med 2003;31(5):1312-7. 